\title{
Long-Term Maintenance of Organizational Strategies in Kindergarten Children
}

\author{
Martha CARR \\ University of Georgia \\ AND \\ WolfGang SCHNEIDER \\ Max Planck Institute for Psychological Research, Munich
}

\begin{abstract}
The goal of the present study was to determine whether 4- and 5-year-old kindergarten children could he trained to maintain an organizational strategy over ?. and 8-week periods through an elaborate training program. A second goal was to assess the effects of the training program on strategy awareness. Twenty-eight kindergarten children were pretested on two sort-recall tasks and their awareness of the use of the clustering strategy was assessed through a protocol type procedure. Half the children received seven half-hour sessions of individual training in the clustering strategy and half the children participated in a control group. Both groups were post-tested on two sort-recall tasks 2 weeks following training and again 8 weeks following training. Strategy awareness, as measured by verbal protocol, was assessed at both post-test points. The elaborate strategy training program was successful in inducing short- and long-term strategy maintenance of the clustering strategy. Trained children's clustering during sorting and clustering during recall was consistently related to the amount of items correctly recalled. No differences in strategy awareness were found. These findings demonstrate that the elaborate training procedure used in this study can be a very effective memory technique for young kindergarten children. 1991 Academic Press, Inc.
\end{abstract}

Recent studies of strategy use in young, preschool age children indicate that young children are capable of deliberate strategy use (Wellman, 1988) and that young children possess knowledge about memory strategies (Yussen \& Bird, 1979; Yussen, Levin, Berman, \& Palm, 1979). Children as young as 2 and 3 years of age will attempt to improve recall by attending to to-be-remembered items (Baker-Ward, Ornstein, \& Holden, 1984; DeLoache, Cassidy, \& Brown, 1985) and by using physical cues for recall (e.g., Heisel \& Ritter, 1981). Furthermore, young children can transfer a strategy if they are familiar with the task items (Brown, Kane, \& Long, in press).

Training studies have gone a step further than developmental studies by

We are grateful to Elizabeth Kulczar for her assistance in collecting the data. Reprint requests should be sent to Martha Carr, Department of Educational Psychology, 325 Aderhold Hall, University of Georgia, Athens, GA 30602. 
investigating the reactions of children of different ages and skill levels to strategy training. The results of training studies indicate that it is knowledge, not age, that constrains strategy use. For example, 7- and 8-year-old children can be trained to use an organizational strategy resulting in better clustering, recall, and task-related metamemory (Paris, Newman, \& McVey, 1982). In addition, training studies have indicated that even children as young as 3 years can learn and benefit from the prompted use of relatively sophisticated memory strategies such as elaboration (Pressley \& Mac Fadyen, 1983; Pressley, Samuel, Hershey, Bishop, \& Dickenson, 1981) or a clustering strategy (Moely, Olsen, Halwes, \& Flavell, 1969), and that prompted strategy use in young children is accompanicd by improved performance (Pressley et al., 1981).

Simply training a strategy, however, does not guarantee its continued use beyond the actual training session (e.g., Keeney, Cannizzo, \& Flavell, 1967). A lack of metacognitive knowledge, which is believed to be necessary for the unprompted use of a strategy, is one probable reason why many training programs have failed to produce maintenance of strategies (Brown, 1978; Flavell, 1978). Given that even 3-year-olds possess some prerequisite metacognitive knowledge about variables that affect memory performance (Wellman, 1977), and metamemory is related to strategy use and superior memory performance (Greulich \& Baker-Ward, 1989; Schneider \& Sodian, 1988), training programs that include metacognitive components should result in strategy maintenance.

The lack of organizational knowledge base of young children is another reason for their failure to use and maintain strategies. Young children often fail to recognize and use meaningful connections within tobe-remembered information (Lange, 1973; Frankel \& Rollins, 1982). When young children organize information it is typically according to its degree of associativity as opposed to typicality (Bjorklund, 1985; Frankel \& Rollins, 1985). As a result, young children's recall is poorer than that of older children or adults (Frankel \& Rollins, 1985). Maintenance of an organizational strategy, therefore, will be dependent on training children to search for meaningful associative and categorical connections within the to-be-remembered information and to use this organization at recall.

To date, only a few studies have attempted to train a strategy for maintenance in young children and none have explored long-term maintenance of a strategy. The purpose of the present study was to determine whether 4- and 5-year-old kindergarten children could be trained to maintain an organizational strategy over 2- and 8-week periods through an elaborate training program. Since strategy maintenance seems to be closely tied to metacognitive awareness, a second goal was to assess the effects of the training program on strategy awareness as well as the relationship between strategy awareness and clustering. 
In the present study, 28 kindergarten children were pretested on two sort-recall tasks and then randomly assigned to either a trained or a control group. Children in the trained group received seven half-hour sessions of intensive strategy training on a clustering strategy. The training included information about how and why the strategy could be applied as well as opportunities for feedback about clustering and recall performance. Children in the control group were given the training materials but received no instruction. Two weeks and eight weeks after the last training session all children were post-tested on two sort-recall tasks. Strategy awareness was assessed by means of verbal protocols at pretest and both post-tests. It was expected that an enriched training program would promote both maintenance of the clustering strategy and awareness of the strategy in the form of increased reports of clustering.

\section{METHOD}

\section{Subjects}

Twenty-eight children attending a kindergarten in Munich, West Germany, participated in the present study. The average age of the children in months was $M=59.00(S D=3,88)$.

\section{Design}

The children were individually pretested on two sort-recall tasks. During the sorting procedure children's awareness of the clustering strategy was assessed through a protocol type procedure. Following this, the children were randomly assigned to either a control or a treatment condition. Children in the training condition received seven half-hour sessions of individual training in the clustering strategy using sets of items that were of varying number and associativity. Control children received the same materials as the trained children and worked with these materials for the same amount of time but were given no instruction of any kind. Both trained and control group children were post-tested on two sort-recall tasks 2 weeks following training and again 8 weeks following training. Strategy awareness, as measured by verbal protocol, was assessed at both post-test points.

\section{Procedure and Materials}

Each child was seen individually in a quiet room in the kindergarten. At the beginning of the pretesting session, the experimenter (a 27 -year-old research assistant) introduced herself to each child and explained that the study she was doing was about how children remember things. Next, the researcher explained that the child would be given some pictures and that he or she could do anything he or she wanted to do with the pictures in order to remember them for later recall. In addition, it was explained that in order to best help the researcher understand how children remember he or she should verbalize his or her thoughts during the sorting and study period. A verbal protocol, as opposed to a questionnaire or interview method, was used to assess strategy awareness because it allowed for a measure of "on line" strategy awareness and lessened the likelihood of problems related to the child's inability to interpret hypothetical questions or to make retrospective reports. Following this, the researcher gave the child the first of two sets of clusterable items. One set of six pictures which had been mounted on a cardboard backing $(4 \times 4 \mathrm{~cm})$ was composed of multiple geometric shapes that could be clustered on the basis of color (red and blue). A second set of six pictures of similar size were clusterable into a toys group (rocking horse, ball, drum) 
and a clothes group (jacket, pants, socks). These simple, easily clusterable items were chosen for the pretest in order to avoid a floor effect for the 4- and 5-year-old children. The clustering tasks were randomly presented. After the children were given the pictures they were told that they would be given a short time $(2 \mathrm{~min})$ to study the pictures. Each child's verbal protocol was taped for later scoring. Following the study period, the researcher photographed the picture arrangement and collected the pictures. The children were then asked to recall the pictures. When the children had stopped recalling the researcher prompted them by asking "Do you remember anything else?" Clustering during sorting and recall was calculated using the adjusted ratio of clustering (ARC) score formula (Roenker, Thompson, \& Brown, 1971). ARC scores are advantageous because they allow a measure of clustering independent of set size and number of categories used. ARC scores for the two pretest sets were averaged for a pretest clustering during sorting score. A pretest clustering during recall score was similarly constructed. I ikewise, percentage recall was assessed for each sort-recall task and averaged for a pretest percentage recall score. Strategy awareness at pretest, post-test 1 , and post-test 2 was scored as follows: Two points were given if the child mentioned the grouping of the items either explicitly or implicitly for both tasks (e.g., blue things at the bottom, red things at the top); 1 point was given if grouping was mentioned for only one of the tasks; 0 points were given for no mention of grouping of either task.

Organizational strategy training consisted of seven half-hour, sessions taking place every other school day over a 3-week period. The children were trained individually. During each training session the children were given the opportunity to work with the clustering strategy using two sort-recall tasks. The procedure for the training sessions was identical to that of the pretest session except that feedback was given about the accuracy of their clustering and recall performance. The instructor explained how recall was affected by the child's use (or lack of use) of the clustering strategy (e.g., "You remembered more this time because you sorted the pictures out so that they went together in this way."). In addition, the instructor explained how and why poorly clustered items could be belter clustered by pointing out three similarities between the items and the group to which they belong (e.g., "These three are pictures of plants. They all grow, they all have petals or leaves, and they all need water and dirt to live. So, let's move them together."). Three reasons were given for the inclusion of a set of items in a group in order to produce a deeper understanding of the relationship between categorizable items by the children. Appropriate clusterings were similarly pointed out and reinforced. Following this, the children were given a second opportunity to work with the clustering strategy. As with the first task, after the children had clustered and recalled, the instructor reviewed the accuracy of both the clustering and the recall, made suggestions for better clustering, and reinforced the relationship between clustering and recall performance. If a child inappropriately clustered items, the instructor explained how and why the child's clustering did not produce agreement between the item and the category.

Nontypical items and items of low associativity were included in the training program since young children have particular trouble with these items (Frankel \& Rollins, 1985). The clusterable items used during training were constructed to be of varying number, associativity, and typicality (Marshall \& Coffer, 1970). For the first two training sessions the associativity and typicality level of the pictures was high (e.g., furniture: chair, table, bed, sofa). Beginning with the third training session the difficulty of the clustering problems in terms of associativity and typicality varied from high to low associativity and typicality. This was done so that the child would have an opportunity to practice clustering at many levels of difficulty. For example, in session 5 one sort-recall task included items that could be categorized as vegetables (carrots, beans, lettuce), wild animals (zebra, fox, seal), and buildings (hut, castle, school). The number of items per clustering group varied from two to six and the number of clustering groups per sort-recall task was varied between two and 
four. This was done in order to discourage children from using clustering group size or sort-recall set size as a criterion for grouping. The control group received identical materials except they did not receive clustering training. Control group children spent the same amount of time on task as the training group. Instead of receiving training these children were told to work with the items in any way that they wished.

Post-test 1 . Two weeks following the last training session, training and control group children were individually tested on two sort-recall tasks. The items in the first sort-recall task consisted of 15 pictures of items clusterable into three sets of 4 items (animals, vehicles, fruit) and one set of 3 items (clothes). The second sort-recall task consisted of 15 items clusterable into three groups with 5 items per group (furniture, tools, playground equipment). These items were of low associativity and typicality; for example, the animal group included an eagle, a hippo, a goat, and a whale and therefore should be a good test of the effectiveness of the training program. Although the use of different test items from pretest to post-test 1 to post-test 2 disallowed a test of intraindividual change, it allowed for a more powerful test of the "trainability" of the clustering strategy.

Post-test 2. Eight weeks following the last training session children in both the treatment and the control groups were individually tested for clustering during sorting, clustering during recall, percentage recall, and strategy awareness. For this post-test, one sort-recall task consisted of 17 items clusterable into one set of 6 items (animals), one set of 5 items (food), one set of 4 items (tools), and one set of 3 items (vehicles). The second sort-recall task was composed of 15 items of low associativity and typicality which were clusterable into one set of 5 items (animals), two sets of 4 items (body parts, things that make light), and one set of 2 items (plants). For both post-tests the two sort-recall tasks were randomly administered. The procedure and scoring for clustering during sorting, clustering during recall, percentage recall, and strategy awareness for the post-test 1 and post-test 2 were identical to those of the pretest. It should be noted that, although some of the categories were repeated throughout training and testing, the items within the categories changed from task to task. Some previously used items were reused; however, these items were always matched with new and different category items.

\section{RESULTS}

Means and standard deviations for percentage recall, clustering during sorting, clustering during recall, and strategy awareness for the training and control groups are presented in Table 1. One goal of this strategy training study was to determine whether the treatment program improved performance, in this case, recall. To this point, a 2 (group) $\times 2$ (time of testing) repeated measures analysis of covariance was performed on percentage recall using recall at pretest as the covariate. Children in the trained group recalled more items than control children $(F(1,25)=22.91$, $p<.01)$. Planned comparisons based on the data of the second post-test, conducted at the $p<.01$ level, were carried out to assess long-term effects of the training program. Because of the small sample size, posttest 2 mean differences were calculated using separate variance estimates (Winer, 1971). The long-term effects of training on recall proved to be significant, $t(23.91)=-4.58$. There was no effect for sessions and no significant interaction. 


\section{TABLE 1}

Means and Standard Deviations (in Parentheses) for Percentage Recall, Clustering during Sorting, Clustering during ReCall, and Strategy AWARENESS

\begin{tabular}{lcc}
\hline \multirow{2}{*}{ Variable } & \multicolumn{2}{c}{ Group } \\
\cline { 2 - 3 } \multicolumn{1}{c}{ Control } & Trained \\
\hline Pretest, percentage recall & $73.71(18.43)$ & $73.68(20.16)$ \\
Post-test 1, percentage recall & $40.32(13.02)$ & $62.79(22.49)$ \\
Post-test 2, percentage recall & $34.21(15.39)$ & $65.93(20.86)$ \\
Pretest, clustering (sort) & $.13(.43)$ & $.22(.64)$ \\
Post-test 1, clustering (sort) & $.17(.32)$ & $.73(.23)$ \\
Post-test 2, clustering (sort) & $.07(.13)$ & $.78(.29)$ \\
Pretest, clustering (recall) & $.22(.31)$ & $.31(.59)$ \\
Post-test 1, clustering (recall) & $.37(.30)$ & $.78(.29)$ \\
Post-test 2, clustering (recall) & $.34(.52)$ & $.85(.28)$ \\
Pretest, strategy awareness & $.21(.43)$ & $.21(.43)$ \\
Post-test 1, strategy awareness & $.14(.36)$ & $.21(.43)$ \\
Post-test 2, strategy awareness & $.21(.43)$ & $.50(.86)$ \\
\hline
\end{tabular}

\section{Clustering during Sorting}

If the superior performance of the trained group was due to the use of clustering during sorting then trained children should show a similar superiority in the use of the clustering strategy during sorting. A 2(group) $\times$ 2(time of testing) repeated measures ANCOVA performed on clustering during sorting using pretest clustering during sorting as the covariate indicated that trained children's clustering during sorting was superior to that of the control group children, $F(1,25)=94.46, p<.01$. Planned comparisons of the second post-test clustering during sorting data indicated significantly more clustering during sorting by the trained group at post-test $2, t(17.88)=-8.41$, at the $p<.01$ level. Neither the interaction nor the main effect for sessions was significant.

\section{Clustering during Recall}

If the use of an organizational strategy is responsible for improved recall performance, clustering during recall should show a corresponding improvement in the trained group but not in the control group. A 2(group) $\times 2$ (time of testing) repeated measures ANCOVA on clustering during recall using clustering during recall pretest as the covariate indicated that the trained group clustered significantly more than the control group during recall, $F(1,25)=20.27, p<.01$. Planned comparisons based on the clustering during recall data at post-test 2 showed that the trained group maintained its superior clustering during recall at long-term follow-up, $t(19.75)=-3.28$ at the $p<.01$ level. Neither the main effect for sessions 
nor the interaction was significant. Intercorrelations among clustering and recall measures were calculated to explore the interrelationships among clustering and recall measures in the pretest and post-test sessions. Results are given in Table 2. As can be seen in Table 2, the intercorrelations among clustering and recall measures at pretest were mostly nonsignificant for both groups, indicating that individual differences in sorting behavior were not related to individual differences in recall. Effects of the training procedure are reflected in the different pattern of results for the trained group. Here, clustering during sorting was significantly related to recall at both post-tests. Moreover, significant intercorrelations among clustering during sorting and clustering during recall as well as clustering during recall and recall were obtained for the trained children at the second post-test. These findings indicate that the trained strategy influenced both the organization of recall and the amount of information correctly recalled. This was generally not true for the control children, with the only exception being that those children who sorted the items were also those who recalled the items best.

\section{Strategy Awareness}

Likewise, since metacognition is believed to play an important role in strategy use (Pressley, Borkowski, \& Schneider, 1987), and since the training included metacognitive information about the applicability of the strategy, it was expected that the superior strategy use of the trained group would be accompanied by superior strategy awareness. No group

TABLE 2

Intercorrelations among Clustering during Sorting, Clustering during Recall, and Recall, as a Function of Measurement Point (Coefficients for Control Children in Parentheses)

(2) Clustering (sort)

(3) Clustering (recall)

(a) Pretest

(1) Recall

$.06(-.01)$

$-.14(.16)$

(2) Clustering (sort)

$.11(.52)^{*}$

(3) Clustering (recall)

(b) Post-test 1

(1) Recall

(2) Clustering (sort)

(3) Clustering (recall)

$.63 *(.18)$

$.35 \quad(.05)$

$.26(-.08)$

(c) Post-test 2

(1) Recall

(2) Clustering (sort)

(3) Clustering (recall)

$.63 *(-.20)$

$.57 *(.27)$

Note. Asterisks denote coefficients significant at the $p=.05$ level. 
differences were found, however, between trained and control children despite apparent differences in their means (cf. Table 1).

Strategy awareness correlated with the use of the clustering during sorting only at pretest for the trained and control groups, $r=.51, p<.05$ and $r=.59, p<.05$, respectively; and at post-test 1 for the control group, $r=.50, p<.05$. Strategy awareness also correlated with percentage recall for the control group at post-test $2, r=.67, p<.01$.

It should be noted, however, that these correlations may be misleading because only few children explicitly mentioned grouping strategies while studying the items. That is, only 3 out of 14 subjects in both groups mentioned grouping at pretest, and only 4 children of the control group referred to grouping at the second post-test. It appears, then, that the success of training reflected in the clustering and recall scores did not substantialize in the strategy awareness measure.

\section{DISCUSSION}

The main purpose of this study was to assess whether 4- and 5-year-old kindergarten children can be trained to maintain an organizational strategy over 2- and 8-week periods. The results indicate that the elaborate strategy training program was successful in inducing short- and long-term strategy maintenance: In both post-tests, children in the trained group conceptually organized the items significantly better than the control children, whose clustering score remained at chance level. Even more importantly, both trained children's clustering during sorting and clustering during recall was consistently related to the amount of items correctly recalled. A comparison of recall performance in the experimental and control group reveals that while there were no group differences at pretest, trained children recalled significantly more than the control children at both post-tests. Taken together, these findings demonstrate that the elaborated training procedure used in this study was very effective in young kindergarten children; trained subjects were able to maintain superior performance even after an 8-week period without any additional prompting.

The results of our study indicate that young children do not spontaneously use organizational strategies, as demonstrated by the low clustering scores at pretest. This finding is at odds with the results of an experimental study by Sodian, Schneider, and Perlmutter (1986) who compared the effects of a "play-and-remember" and a "sort-and-remember" instruction on 4- and 6-year-old's performances in a sort-recall task. Sodian et al. (1986) reported significant levels of clustering during sorting as well as high levels of clustering during recall for the children in the sortand-remember condition who were instructed to put all those items together that go together. The discrepancy between our findings and those 
obtained by Sodian et al. (1986) may be due to the fact that our instruction at pretest did not include any sorting cue; children were simply encouraged to do something with the items. Our results confirm those reported by Moely et al. (1969) and provide further evidence for the production deficiency hypothesis proposed by these authors. That is, our findings show that kindergarten children do possess conceptual skills but do not bring these skills into play spontaneously as a means of coping with the sortrecall task. The fact that kindergarten children using the organizational strategy after training benefit from its use clearly indicates that they are "production-deficient" and not "mediation-deficient." If the latter were true, the instruction of organizational strategies should improve strategy use but not recall.

It might be argued that the improved performance of the trained group in clustering and recall could be due, not to the training program, but to repeated experiences with the memory task. To the best of our knowledge no studies that directly measure the effects of repeated recall trials on recall performance have been done. Nevertheless, since the recall and strategy use of young children is typically very poor (e.g., Myers \& Perlmutter, 1978; Perlmutter \& Myers, 1979) and improvement in recall performance seems to be highly dependent on the use of encoding and retrieval cues (Schneider \& Pressley, 1989), it is unlikely that such a significant improvement in recall and clustering was caused by the recall experience itself.

One basic assumption of this study was that metacognitive knowledge is necessary for the unprompted use of a strategy. As our training program included information about how and why an organizational strategy can be useful, we expected higher strategy awareness for our trained subjects, as compared to the control children. This expectation was not confirmed. Only a small number of children in both groups who already mentioned grouping as a strategy at pretest continued to do so during the post-test sessions. Please note that the significant and apparently substantial correlations between sorting at pretest and strategy awareness found for both groups were due to the fact that the three children who grouped the items into semantic categories also stated explicitly that they were grouping; the majority of children did not group the items and consequently did not mention grouping strategies. This shows that the correlations were considerably influenced by extreme scores in the bivariate distribution which, in the present study, obscured the "true" relationship.

Unexpectedly, most of our trained children who grouped items into categories did not mention grouping as a strategy. There are two possible explanations for this phenomenon: First, the metacognitive information embedded in the training procedure was not acquired by our subjects. 
That is, children only learned how to use the clustering strategy but not why it is important to use it. Alternatively, it could be that the trained children indeed acquired some metacognitive knowledge but that our awareness measure was not sensitive enough to assess this knowledge.

Although it is impossible to decide on these alternative explanations on the basis of the available data, two findings indicate that the latter assumption may be true. First, trained children were able to use the organizational strategy regardless of task difficulty. Although typicality and associativity were not systematically varied, the low associativity and typicality of many of the items did not appear to affect clustering as would be expected. Usually, the presence of low associations among items and low item typicality negatively affects clustering even in young gradeschool children. The fact that older elementary school children show greater flexibility has been interpreted as a sign of metacognitive awareness, that is, deliberate decisions about strategy use (cf. Schneider, 1986).

Second, as has been pointed out by Frankel and Rollins (1985), deliberate strategy use may be reflected by significant intercorrelations between clustering during sorting and clustering during recall on the one hand, and between clustering and recall on the other hand. A closer look at Table 2 reveals that intercorrelations among these measures were indeed high for our trained subjects, particularly at the second post-test. We are thus inclined to believe that our trained subjects had an implicit understanding about the utility of organizational strategies but that this implicit understanding had not solidified into explicit verbalized knowledge that could be inferred from the strategy awareness measure. The problem remains to come up with a metamemory instrument suited to assess taskspecific metacognitive knowledge of young children.

All in all, our findings demonstrate that kindergarten children do not spontaneously use organizational strategies in sort-recall tasks. While young children are capable of deliberate strategy use in familiar memory situations and also seem to know about the advantages of memory strategies in such situations (cf. Schneider \& Sodian, 1988; Wellman, 1988), the use of organization as a memory strategy does not develop before the elementary school years. The good news is that organizational strategies can be trained effectively even in kindergarten children. In our view, the success of the training procedure used in this study was mainly due to three procedures identified as major determinants of strategy acquisition (cf. Waters \& Andreassen, 1983): Manipulations of task structure and procedure, task familiarization and practice, and explicit verbal instruction. Further investigations into the strategy acquisition process in young children should not only focus on strategy maintenance but also address the issue of strategy generalization in order to explore the possibilities and limitations of this training approach. 


\section{REFERENCES}

Baker-Ward, L.. Ornstein, P. A., \& Holden, D. J. (1984). The expression of memorization in early childhood. Journal of Experimental Child Psychology, 37, 555-575.

BJoRKLUND, D. F. (1985). Associative and categorical processed in children's memory: The role of automaticity in the development of organization in free recall. Journal of Experimental Child Psychology, 39, 599-617.

BRown, A. L. (1978). Knowing when, where, and how to remember: A problem of metacognition. In R. Glaser (Ed.), Advances in instructional psychology (pp. 77-165). Hillsdale, NJ: Lawrence Erlbaum.

Brown, A. L., Kane, M. J., \& Long, C. (in press). Analogical transfer in young children: Analogies as tools for communication and exposition. Applied Cognitive Psychology.

Deloache, J. S., Cassidy, D. J., \& Brown, A. L. (1985). Precursors of mnemonic strategies in very young children's memory. Child Development, 56, 125-137.

Flavell, J. H. (1978). Metacognitive development. In J. M. Scandura \& C. J. Brainerd (Eds.), Structurallprocess theories of complex human behavior (pp. 213-246). Alpen a.d. Rijn: Sijhoff \& Nordhoff.

Frankel, M. T., \& Rollins, H. A., JR. (1982). Age related differences in clustering: A new approach. Journal of Experimental Child Psychology, 34, 113-122.

Frankel, M. T., \& Rollins, H. A., JR. (1985). Associative and categorical hypotheses of organization in free recall of adults and children. Journal of Experimental Child Psychology, 40, 304-318.

Greulich, E. D., \& Baker-Ward, L. (1989, April) The effects of strategic and metamemorial training on kindergarten children's memory and metamemory ability. Paper presented at the biennial meeting of the Society for Research in Child Development, Kansas City, MO.

Heisel, B. E., \& RitTeR, K. (1981). Young children's storage behavior in a memory for location task. Journal of Experimental Child Psychology, 31, 250-364.

Keeney, J. J., Cannizzo, S. R., \& Flavell, J. H. (1967). Spontaneous and induced verbal rehearsal in a recall task. Child Development, 38, 953-966.

LANGE, G. (1973). The development of conceptual and rote recall skills among school age children. Journal of Experimental Child Psychology, 15, 394-407.

Marshall, G. R., \& Coffer, C. N. (1970). Single-word free-association norms for 328 responses from the Connecticut Cultural Norms for verbal items in categories. In L. Postman \& G. Keppel (Eds.), Norms of word associations (pp. 321-360). New York: Academic Press.

Moely, B. E., Olson, F. A., Halwes, T. G., \& Flavell, J. H. (1969). Production deficiency in young children's clustering recall, Developmental Psychology, 1, 26-34.

Myers, N. A., \& Perlmutter, M. (1978). Memory in the years two to five. In P. A. Ornstein (Ed.), Memory development in children (pp. 191-218). Hillsdale, NJ: Erlbaum.

Paris, S. G., Newman, R. S., \& MCVEY, K. A. (1982). Learning the functional significance of mnemonic actions: A microgenetic study of strategy acquisition. Journal of Experimental Child Psychology, 34, 490-509.

Perlmutter, M., \& Myers, N. A. (1979). Development of recall in 2- to 4-year-old children. Developmental Psychology, 15, 78-83.

Pressley, M., Borkowski, J. G., \& Schneider, W. (1987). The cognitive strategy user: Coordinating metacognition, strategy use, and knowledge. In R. Vasta and G. Whitehurst (Eds.), Annals of child development, (Vol. 4, pp. 80-129). Greenwich, CT: JAI Press.

Pressley, M., \& Mac Fadyen, J. (1983). Mnemonic mediator retrieval at testing by preschool and kindergarten children. Child Development, 54, 474479.

Pressley, M., Samuel, J., Hershey, M. M., Bishop, S. L., \& Dickinson, D. (1981). Use 
of a mnemonic technique to teach young children foreign language vocabulary. Contemporary Educational Psychology, 6, 110-116.

Roenker, D. L., Thompson, C. P., \& Brown, S. (1971). Comparison of measures for the estimation of clustering in free recall. Psychological Bulletin, 76, 45-48.

SCHNEIDER, W. (1986). The role of conceptual knowledge and metamemory in the development of organizational processes in memory. Journal of Experimental Child Psychology, 42, 218-236.

Schneider, W., \& Pressley, M. (1989). Memory development between 2 and 20. New York: Springer-Verlag.

SChNEIDER, W., \& Sodian, B. (1988). Metamemory-memory behavior relationships in young children: Evidence from a memory-for-location task. Journal of Experimental Child Psychology, 45, 209-233.

Sodinn, B., Schneider, W., \& Perlmutter, M. (1986). Recall, clustering, and metamemory in young children. Journal of Experimental Child Psychology, 41, 395-410.

WATERs, H. S., \& ANDreassen, C. (1983). Children's use of memory strategies under instruction. In M. Pressley \& J. R. Levin (Eds.), Cognitive strategy research: Psychology foundations (pp. 3-24). New York: Springer-Verlag.

Wellman, H. M. (1977). Preschooler's understanding of memory-relevant variables. Child Development, 48, 1720-1723.

Wellman, H. M. (1988). The early development of memory strategies. In E. F. Weinert \& M. Perlmutter (Eds.), Memory development: Universal changes and individual differences (pp. 3-29). Hillsdale, NJ: Erlbaum.

WINER, B. J. (1971). Statistical principles in experimental design, 2nd Edition. New York: McGraw-Hill.

YUSSEN, S. R., \& BrRD, J. E. (1979). The development of metacognitive awareness in memory, communication, and attention. Journal of Experimental Child Psychology, 28, 300-313.

Yussen, S. R., Levin, J. R., Berman, L., \& Palm, J. (1979). Developmental changes in the awareness of memory benefits associated with different types of picture organization. Developmental Psychology, 15, 447-449. 